\title{
Design Considerations for Dispersion Control with a Compact Bonded Grism Stretcher for Broadband Pulse Amplification
}

\author{
H. Kiriyama, ${ }^{1}$ H. Sasao, ${ }^{1}$ A. Sugiyama, ${ }^{1}$ and K. Ertel ${ }^{2}$ \\ ${ }^{1}$ Kansai Photon Science Institute, Japan Atomic Energy Agency, 8-1-7 Umemidai, Kizugawa 619-0215, Japan \\ ${ }^{2}$ Central Laser Facility, STFC Rutherford Appleton Laboratory, Chilton, Didcot OX11 OQX, UK \\ Correspondence should be addressed to H. Sasao, sasao.hajime@jaea.go.jp
}

Received 27 March 2012; Accepted 13 May 2012

Academic Editors: A. Bogoni, D. Y. Choi, Y. Leng, V. Matejec, D. Monzon-Hernandez, C. Shu, and A. A. Sukhorukov

Copyright ( $) 2012$ H. Kiriyama et al. This is an open access article distributed under the Creative Commons Attribution License, which permits unrestricted use, distribution, and reproduction in any medium, provided the original work is properly cited.

\begin{abstract}
We report on the design of a compact grism-pair stretcher for a near-infrared noncollinear optical parametric chirped-pulse amplification (OPCPA) system. The grisms are produced by bonding a grating to a prism using a resin. The stretcher is capable of controlling a bandwidth of over $300 \mathrm{~nm}$, which is suitable for parametric amplification of few-cycle pulses. After amplification, pulses can be compressed by the dispersion of optical glass, and the residual group-delay can be compensated with an acousto-optic programmable dispersive filter (AOPDF).
\end{abstract}

\section{Introduction}

Energetic few-cycle pulses enable many new applications in attosecond science, quantum coherent control, and nonlinear optics [1]. Single attosecond XUV pulses can be produced via high harmonic generation (HHG) driven by few-cycle pulses, enabling attosecond spectroscopic applications and the control of atomic-scale electron motion [2]. Techniques for generating few-cycle pulses, such as, the use of a carefully dispersion managed Kerr-lens mode-locked Ti:sapphire oscillator [3], self-phase modulation in a single-mode fiber [4], and in a gas-filled capillary [5] have been proposed and demonstrated. However, it has been difficult to increase the energy of the few-cycle pulses beyond the few-mJ level [6]. Presently, noncollinear optical parametric chirped-pulse amplification (OPCPA) is a promising route for synthesis of more energetic few-cycle waveforms $[7,8]$. Similar to classical chirped-pulse-amplification (CPA) [9], generating ultrashort pulses with an OPCPA system involves stretching, amplifying, and subsequently compressing a pulse to its transform limit and requires precise dispersion management [8].

Prism-based compressors compensate only a small amount of dispersion compared to the typical values used in ultra-high-intensity laser systems [10]. To compress a stretched, amplified pulse of a duration of several hundred picoseconds, the required physical dimensions render their use impractical. On the other hand, grating stretchers (compressors) that provide positive (negative) chirp have been widely employed in traditional high-intensity CPA systems [11]. However, a lower grating efficiency in the compressor (typically 50-70\%) results in a considerable energy loss of the amplified pulse. In contrast, a bulk material compressor with positive dispersion offers the advantage of broadband high-transmission efficiency (typically $>90 \%$ ) and facilitates alignment. In order to use an optical glass compressor, a grating-and-prism, so-called grism stretcher is selected as a negative dispersion stretcher that generates the negatively chirped waveform. A grism is a single optical element containing a grating attached to a prism. The grating pair provides most of the pulse stretching, while the prism pair precompensates for third-order dispersion (TOD) in the optical glass compressor. By adjusting parameters, such as, the ratio of TOD to group delay dispersion (GDD), the grism stretcher can be tailored to match that of the bulk compressor. This feature allows dispersion compensation over a wide wavelength range in simple and compact set-up, in contrast to classical prism compressors where this ratio is mostly fixed and has the wrong sign.

A grism pair stretcher that can provide zero TOD was proposed by Tournois [12]. Later, Kane and Squier demonstrated both negative group-delay-dispersion (GDD) and 


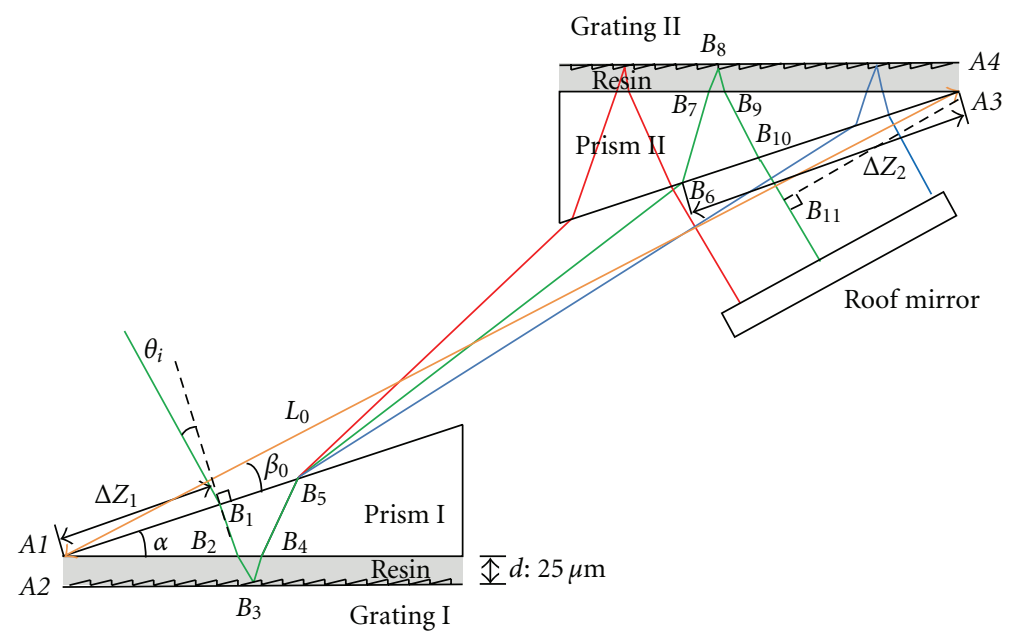

FIGURE 1: Layout for ray tracing in the bonded grism stretcher.

negative TOD with a grism stretcher [13]. In these designs, the transmission gratings were written directly onto the prisms. The negative dispersion of the grism pair stretcher allows the use of transparent bulk material with very high-transmission efficiency for compression. However, the transmission efficiency for this type of grating is low and limits its application in practice. An improved design has been introduced based on a reflective grating which is fabricated directly on the prism surface. The grism stretcher where the gratings and prisms are pressed close together has been designed and tested [14]. In this work we report on the design considerations of a grism pair stretcher where the gratings are bonded to the prisms with a resin. Such "bonded" grisms greatly simplify the fabrication process. We give a comprehensive analysis of the dispersion based on ray tracing. We consider, as an example, the combined design of a grism pair stretcher and optical glass compressor for a fewcycle pulse system that can support a bandwidth in excess of $300 \mathrm{~nm}$ at a center wavelength of $850 \mathrm{~nm}$. We have also successfully fabricated the designed grism. To the best of our knowledge, this is the first grism stretcher that is produced by bonding a grating to a prism using a resin.

\section{Dispersion Analysis with Ray Tracing}

A dispersive device imparts a frequency-dependent phase shift that can be expressed in a Taylor expansion at the central frequency $\omega_{0}$ :

$$
\phi(\omega)=\phi_{0}+\phi_{1}\left(\omega-\omega_{0}\right)+\phi_{2}\left(\omega-\omega_{0}\right)^{2}+\phi_{3}\left(\omega-\omega_{0}\right)^{3}+\cdots,
$$

where the coefficients, $\phi_{n}$ are given by:

$$
\phi_{n}=\left.\frac{1}{n !} \frac{d^{n} \phi(\omega)}{d \omega^{n}}\right|_{\omega_{0}} .
$$

The first order dispersion $\phi_{1}$ describes the group delay (GD). The coefficients $\phi_{2}$ and $\phi_{3}$ represent GDD and TOD, respectively. For propagation through material, GD can be expressed as:

$$
\mathrm{GD}(\lambda)=\frac{1}{c_{o}}\left(n-\frac{\mathrm{d} n}{\mathrm{~d} \lambda}\right) l,
$$

where $\lambda$ is the wavelength, $c_{o}$ the speed of light in vacuum, and $n$ the refractive index. The expression in brackets is also called the group index $N$. For optimum pulse compression in a laser system, the overall GD accumulated in the system has to be constant for all wavelengths in the pulse spectrum. Our bonded grism pair stretcher unit is shown in Figure 1. We have calculated the total group delay in a possible OPCPA system containing a grism stretcher with reflective gratings using the ray-tracing method. The total GD of the stretcher is determined by adding up the GDs experienced along the path lengths (shown as " $B_{1} B_{2}$ " in Figure 1) in the different materials involved. According to the information shown in Figure 1, for two identical prisms the GD at wavelength $\lambda$ can be expressed as:

$$
\begin{aligned}
\mathrm{GD}(\lambda)=2\{ & N_{\text {prism }}\left[B_{1} B_{2}+B_{4} B_{5}+B_{6} B_{7}+B_{9} B_{10}\right] \\
& +N_{\text {resin }}\left[B_{2} B_{3}+B_{3} B_{4}+B_{7} B_{8}+B_{8} B_{9}\right] \\
& \left.+N_{\text {air }}\left[B_{5} B_{6}+B_{10} B_{11}\right]\right\},
\end{aligned}
$$

where $N_{\text {prism }}, N_{\text {resin }}$, and $N_{\text {air }}$ denote the refractive indices of prism, resin, and air, respectively. Instead of $\operatorname{GD}(\lambda), \phi(\omega)$ could be used for the calculation too, but then the additional phase term caused by the diffraction from the gratings needs to be considered too [11]. The factor of 2 accounts for the double pass geometry as shown in Figure 1. The GD for each wavelength can be calculated at a given incident angle by using Snell's law.

\section{Design and Fabrication of the Bonded Grism for Practical Use}

With ray tracing we have modeled and optimized the design of a bonded-grism pair stretcher with broad spectral 
TABLE 1: Optical design parameters.

\begin{tabular}{lcccc}
\hline$\theta_{i}$ degree & $\beta_{0}$ (degree) & $\Delta Z_{1}(\mathrm{~mm})$ & $\Delta Z_{2}(\mathrm{~mm})$ & $L_{0}(\mathrm{~mm})$ \\
\hline 11.5 & 9.42 & 42.1 & 44.3 & 191 \\
\hline
\end{tabular}

TABLE 2: Dispersion terms of the main components at the center wavelength of $850 \mathrm{~nm}$.

\begin{tabular}{lcccc}
\hline & $\begin{array}{c}\text { GDD } \\
{\left[10^{3} \mathrm{fs}^{2}\right]}\end{array}$ & $\begin{array}{c}\text { TOD } \\
{\left[10^{3} \mathrm{fs}^{3}\right]}\end{array}$ & $\begin{array}{c}\text { FOD } \\
{\left[10^{3} \mathrm{fs}^{4}\right]}\end{array}$ & $\begin{array}{c}\text { FOD } \\
{\left[10^{3} \mathrm{fs}^{5}\right]}\end{array}$ \\
\hline Grism pair & -56 & -25.9 & -125 & -202 \\
$\mathrm{TeO}_{2}$ (AOPDF) & 11 & 7.5 & 4.1 & 4.1 \\
$\mathrm{BBO}$ & 0.6 & 0.5 & -0.1 & 0.5 \\
S-TIH53 glass & 46 & 31 & 8.3 & 23 \\
\hline Total & 1.6 & 13.1 & -112.7 & -174.4 \\
\hline
\end{tabular}

acceptance that is required for a broadband OPCPA system to amplify few-cycle pulses. The stretcher gives a negative chirp, which is compensated by the positive chirp in a bulk material (S-TIH53; OHARA) compressor after amplification. In addition, an acousto-optic programmable dispersion filter (AOPDF; Dazzler UWB-600-900, Fastlite), containing a $25 \mathrm{~mm}$ long $\mathrm{TeO}_{2}$ crystal, is inserted after the stretcher for adaptive chirp control. BBO crystals of a total length of $9 \mathrm{~mm}$ provide parametric amplification. For our calculation, the seed pulse is expected to provide radiation with a spectrum extending from $700 \mathrm{~nm}$ to $1000 \mathrm{~nm}$. A $100 \mathrm{ps}$ pump pulse for the OPCPA is assumed, which means that the seed pulse should be stretched to about $50 \mathrm{ps}$ in order to achieve a balance between gain narrowing and conversion efficiency. Thus, the required length of the optical glass compressor is calculated to be $230 \mathrm{~mm}$.

The distance between grating and prism is assumed to be $25 \mu \mathrm{m}$ for both grisms. The gratings are bonded on the prisms with a resin. The refractive index and operational temperature of our resin proposed are 1.55 and up to $100^{\circ} \mathrm{C}$, respectively. The use of the resin permits to mix a quantity of cement and bond many elements before the cement starts to gel. The final design parameters are listed in Table 1, using gratings with 200 lines/mm (C/N020-080-601; SHIMADZU) at a central wavelength of $850 \mathrm{~nm}$. The prisms (N-BAK4; SHOTT) and gratings are commercially available. The apex angle of the prisms $(\alpha)$ is 18.3 degree. The beam path through the stretcher is illustrated in Figure 1. The distance from the apex point of a prism to the position where the beam enters the prism is called the insertion distance $\Delta Z_{1}$ and $\Delta Z_{2}$ for Prism I and Prism II, respectively. $L_{0}$ is the distance between the apexes $A 2$ and $A 3$ of the two prisms. Table 2 lists the dispersion terms of the main dispersive elements of the stretcher-compressor system. Mirrors in the system are not included because their dispersion is negligibly small compared to the main tabulated dispersive elements. The calculated group delay curves of the dispersive components in the laser chain are shown in Figure 2. In our optimal solution, the residual group delay caused by grism stretcher, $\mathrm{TeO}_{2}$ crystal, $\mathrm{BBO}$ crystals, and glass compressor is very well balanced over the full spectrum. The residual

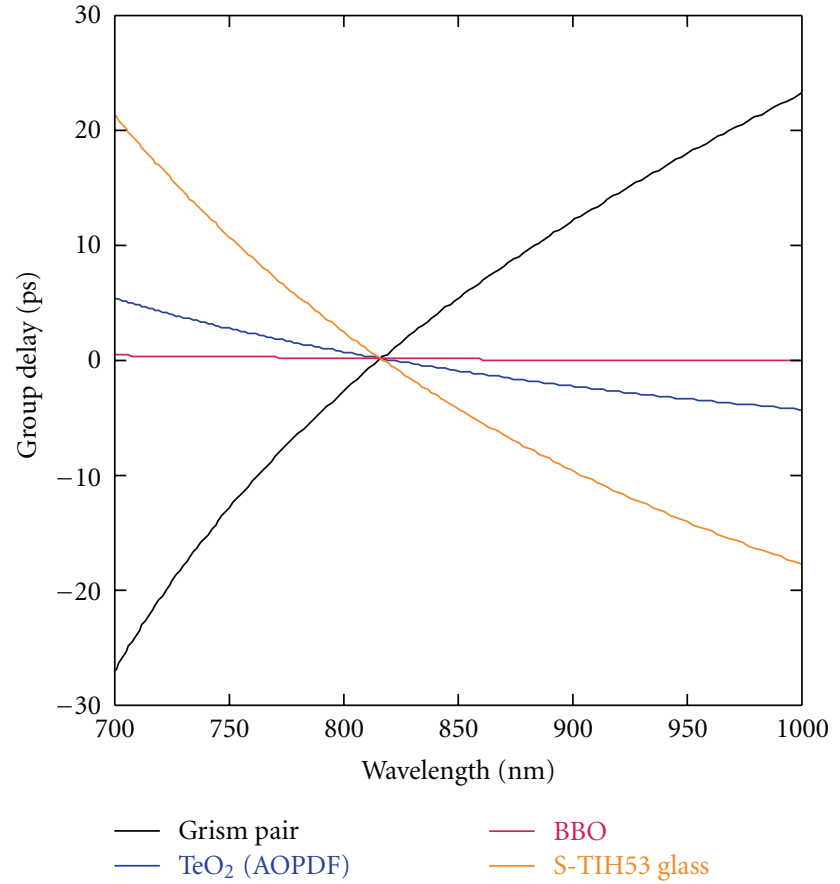

FIGURE 2: Calculated group delay curves of the dispersive components in the system. The black, blue, red, and yellow curves are for the grism stretcher, AOPDF, BBO crystals, and compressor, respectively.

group delay of the chain shown in Figure 3. The shaded parts in the figure show the possible compensation range of the AOPDF. Consequently, residual group delay in the whole spectral range can be compensated by the AOPDF, which has a tuning range of 3 ps, as shown in Figure 3. This will result in a bandwidth-limited pulse that can span a spectrum of $300 \mathrm{~nm}$. In further extending the spectral bandwidth beyond $300 \mathrm{~nm}$, several additional factors have to be considered. The grating with high diffraction efficiency in the spectral range over $300 \mathrm{~nm}$ is required because the higher overall transmission through the grism stretcher improves the contrast of the signal pulse to the parametric fluorescence $[15,16]$. The larger compensation range of the AOPDF is also needed to control the group delay and to obtain shorter pulse duration. A photograph of the bonded grism to be used in an experimental realization, presently in progress, is shown in Figure 4.

\section{Conclusions}

We have presented the design of a stretcher based on bonded grisms, where prisms and gratings are bonded with a resin. We have analyzed its optical performance using optical ray tracing. The performance has been optimized by tuning input parameters, such as, the incident beam angle and prism apex angle. We have designed and fabricated a grism stretcher with a spectral acceptance of up to $300 \mathrm{~nm}$ which is suitable for parametric amplification of few-cycle pulses. The amplified pulse can be compressed by dispersion in bulk material 


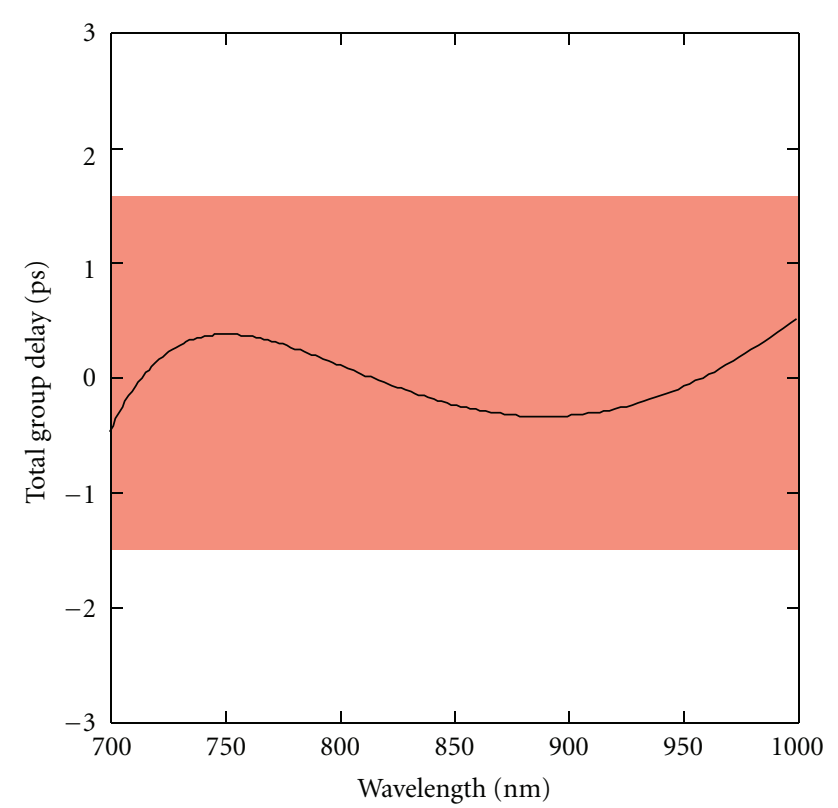

Figure 3: Residual group delay as function of wavelength. The shaded area denotes the compensation range of the AOPDF.

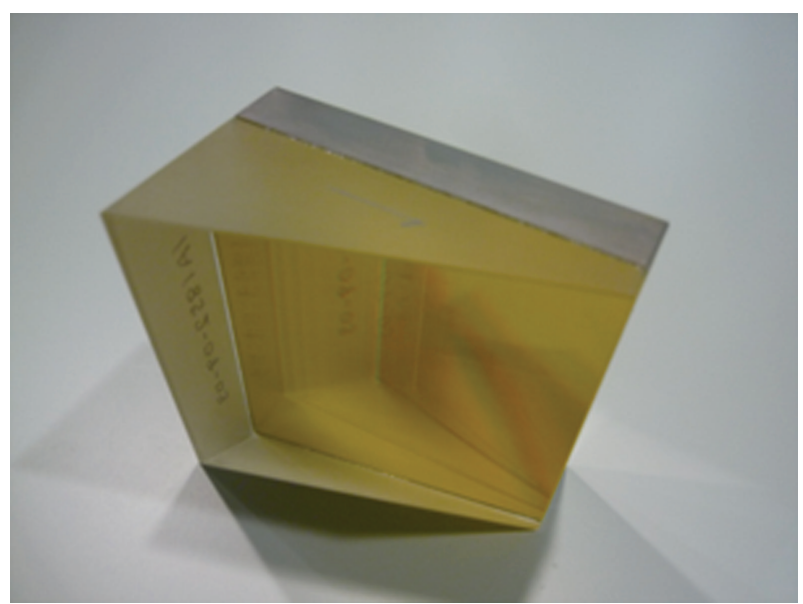

FIGURE 4

which provides high-transmission efficiency. The fabricated grism stretcher is currently being used to stretch the seed pulse from a Ti:sapphire laser oscillator (Rainbow; FEMTOLASERS) with the aim of amplifying a few-cycle pulse.

\section{Acknowledgments}

This work was supported by the "Consortium for Photon Science and Technology" (CPhoST) Program funded by the SCF, MEXT. The authors thank M. Mori, S. V. Bulanov, and P. R. Bolton for helpful discussions.

\section{References}

[1] E. Goulielmakis, M. Schultze, M. Hofstetter et al., "Singlecycle nonlinear optics," Science, vol. 320, no. 5883, pp. 16141617, 2008.

[2] G. D. Tsakiris, K. Eidmann, J. Meyer-ter-Vehn, and F. Krausz, "Route to intense single attosecond pulses," New Journal of Physics, vol. 8, article 19, 2006.

[3] U. Morgner, F. X. Kärtner, S. H. Cho et al., "Sub-two-cycle pulses from a Kerr-lens mode-locked Ti:sapphire laser," Optics Letters, vol. 24, no. 6, pp. 411-413, 1999.

[4] A. Baltuska, Z. Wei, M. S. Pshenichnikov, and D. A. Wiersma, "Optical pulse compression to 5 fs at a $1-\mathrm{MHz}$ repetition rate," Optics Letters, vol. 22, no. 2, pp. 102-104, 1997.

[5] M. Nisoli, S. de Silvestri, O. Svelto et al., "Compression of high-energy laser pulses below 5 fs," Optics Letters, vol. 22, no. 8, pp. 522-524, 1997.

[6] J. H. Sung, J. Y. Park, T. Imran, Y. S. Lee, and C. H. Nam, "Generation of 0.2-TW 5.5-fs optical pulses at $1 \mathrm{kHz}$ using a differentially pumped hollow-fiber chirped-mirror compressor," Applied Physics B, vol. 82, no. 1, pp. 5-8, 2006.

[7] R. T. Zinkstok, S. Witte, W. Hogervorst, and K. S. E. Eikema, "High-power parametric amplification of 11.8-fs laser pulses with carrier-envelope phase control," Optics Letters, vol. 30, no. 1, pp. 78-80, 2005.

[8] F. Tavella, Y. Nomura, L. Veisz, V. Pervak, A. Marcinkevičius, and F. Krausz, "Dispersion management for a sub-10-fs, 10 TW optical parametric chirped-pulse amplifier," Optics Letters, vol. 32, no. 15, pp. 2227-2229, 2007.

[9] D. Strickland and G. Mourou, "Compression of amplified chirped optical pulses," Optics Communications, vol. 56, no. 3, pp. 219-221, 1985.

[10] R. L. Fork, O. E. Martinez, and J. P. Gordon, "Negative dispersion using pairs of prisms," Optics Letters, vol. 9, no. 5, pp. 150-152, 1984.

[11] E. B. Treacy, "Optical pulse compression with diffraction gratings," IEEE Journal of Quantum Electronics, vol. 5, no. 9, pp. 454-458, 1969.

[12] P. Tournois, "New diffraction grating pair with very linear dispersion for laser pulse compression," Electronics Letters, vol. 29, no. 16, pp. 1414-1415, 1993.

[13] S. Kane and J. Squier, "Grating compensation of third-order material dispersion in the normal dispersion regime: sub100-fs chirped-pulse amplification using a fiber stretcher and grating-pair compressor," IEEE Journal of Quantum Electronics, vol. 31, no. 11, pp. 2052-2057, 1995.

[14] T. H. Dou, R. Tautz, X. Gu et al., "Dispersion control with reflection grisms of an ultra-broadband spectrum approaching a full octave," Optics Express, vol. 18, no. 26, pp. 27900 27909, 2010.

[15] H. Kiriyama, M. Mori, Y. Nakai et al., "High temporal and spatial quality petawatt-class Ti:sapphire chirped-pulse amplification laser system," Optics Letters, vol. 35, no. 10, pp. 1497-1499, 2010.

[16] H. Kiriyama, M. Michiaki, Y. Nakai et al., "Highspatiotemporal-quality petawatt-class laser system," Applied Optics, vol. 49, no. 11, pp. 2105-2115, 2010. 

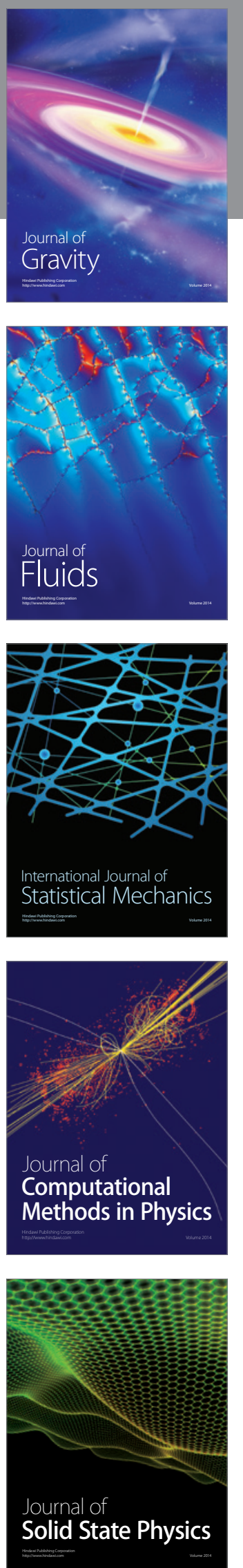
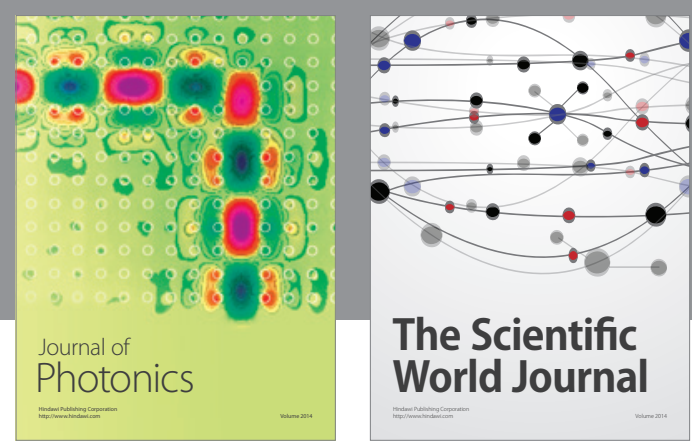

The Scientific World Journal

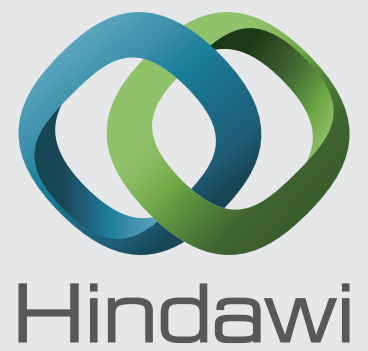

Submit your manuscripts at http://www.hindawi.com
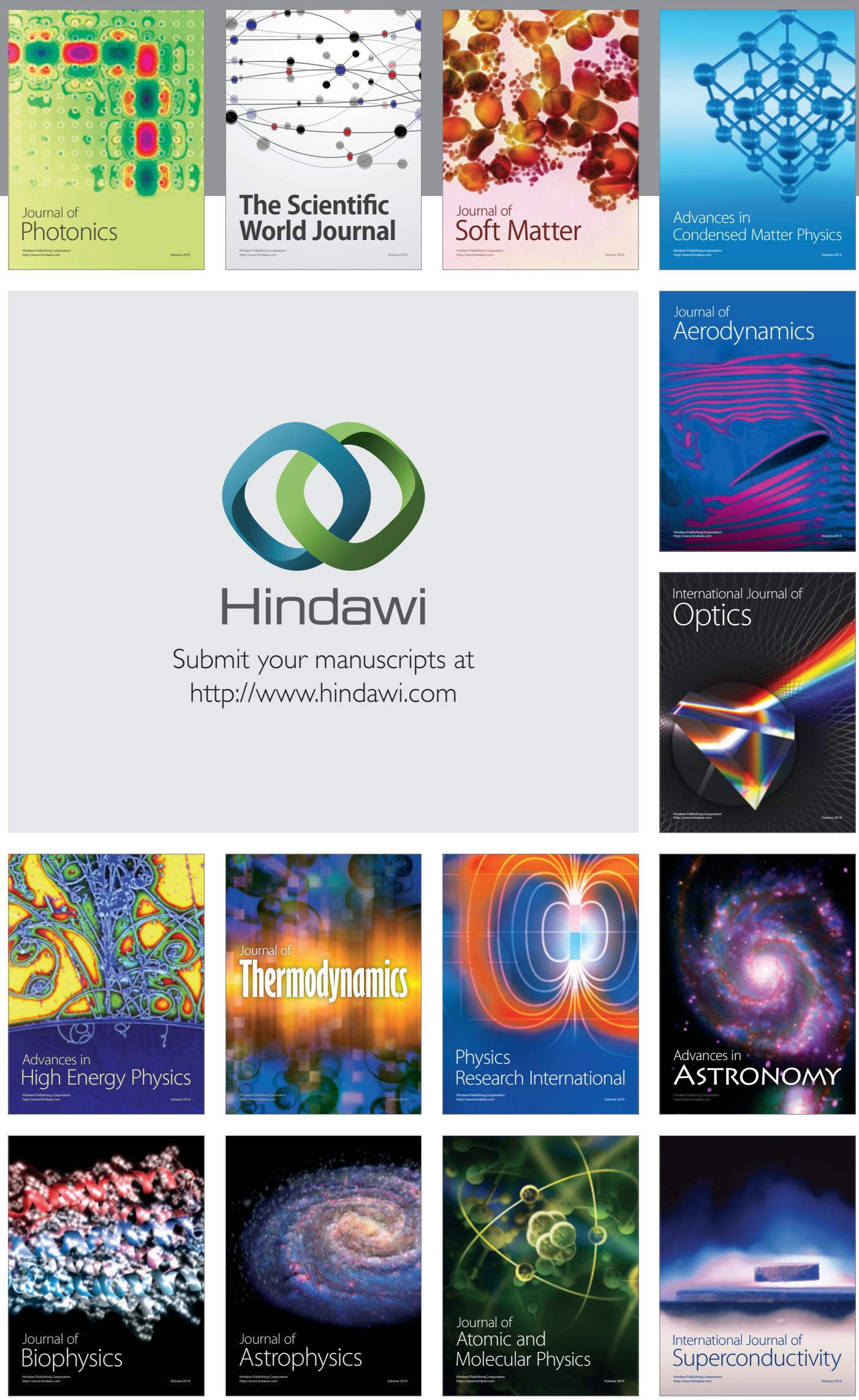
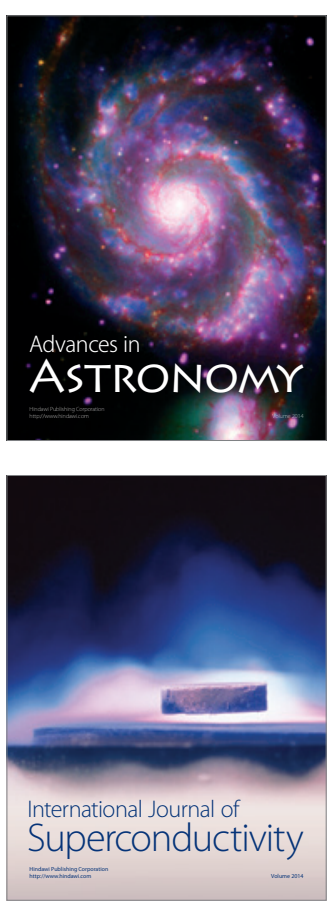